\title{
FLAT SPOTS ON UNIT SPHERES
}

\author{
D. VAN DULST \\ (Received 8 March; revised 18 October 1978) \\ Communicated by A. P. Robertson
}

\begin{abstract}
A flat spot in a Banach space $X$ is an element $x \in S_{X}=\{x \in X:\|x\|=1\}$ with the property that the infimum $m(x)$ of the lengths of all curves in $S_{X}$ joining $x$ to $-x$ is 2 . Flat spots occur in every non-superreflexive space when suitably renormed. A study is made of the geometric implications of the existence of flat spots. Connections with other notions such as differentiability, decomposition constants and Kadec-Klee norms are explored and some renorming results for non-superreflexive spaces are proved.
\end{abstract}

Subject classification (Amer. Math. Soc. (MOS) 1970): 46 B 99.

\section{Introduction and summary}

Let $X$ be a Banach space and let $B_{X}$ and $S_{X}$ denote its unit ball and unit sphere, respectively. Schäffer (1967) introduced a geometric parameter called the girth of $B_{X}$ and defined as follows. For each $x \in S_{X}$ let $m(x)$ be the infimum of the lengths of all curves in $S_{X}$ with initial point $x$ and endpoint $-x$ and let

$$
m(X)=\inf \left\{m(x): x \in S_{X}\right\} .
$$

By reflecting through the origin each curve from $x$ to $-x$, one sees that the number $2 m(X)$ can be regarded as the infimum of the lengths of all centrally symmetric closed curves in $S_{X}$. It is therefore called the girth of $B_{X}$. Always $2 \leqslant m(X) \leqslant 4$ (see Schäffer (1967)) and $m(X)>2$ if and only if $X$ is superreflexive (James and Schäffer (1972)).

If for some $X$ both infima involved in the definition of $m(X)$ are attained and equal 2, that is if there exists a so-called girth curve in $S_{X}$ joining two antipodal points and with length 2 , this has various geometric consequences which justify 
calling such a space flat (Harrell and Karlovitz (1974)). Flat spaces have nonseparable flat duals (Karlovitz (1973)) and cannot be isomorphically embedded into any separable dual space (Harrell and Karlovitz (1974)). Examples are $C[0,1], L^{1}[0,1], L^{\infty}[0,1], l^{\infty}$. If only $m(x)=2$ for some $x \in S_{X}$ (where the infimum may or may not be attained), then $x$ is called a flat spot (Schäffer (1976)). Until recently almost nothing was known about flat spots, not even their existence, except in flat spaces (Schäffer (1976)). In van Dulst and Schäffer (1979) the first examples were given of flat spots in non-flat Banach spaces. Subsequently, Pach (1979) proved that any non-superreflexive Banach space can be equivalently renormed so that it has a flat spot. Since $m(X)>2$ for superreflexive spaces $X$, this completely solves the isomorphic existence problem for flat spots.

In Section 1 we formally state Pach's result for later reference and, for motivation, present probably the simplest example of a non-trivial flat spot. In Section 2 we study the geometry of the unit sphere at a flat spot. More generally, we investigate the geometric implications of the assumption ' $m(x)$ close to 2'. It is shown that in this case there exist two-dimensional subspaces whose unit spheres approximate squares with $x$ as a vertex and with one edge containing $x$ uniformly close to all hyperplanes supporting the unit ball at $x$. We also give estimates for the local moduli of convexity and smoothness at $x$ in terms of $m(x)$. In the limit case $m(x)=2$ both moduli are as bad as can be. It follows in particular that if $x$ is a flat spot then the norm is not Fréchet differentiable and not locally uniformly convex at $x$, and $x$ fails to be a strongly exposed point. We end Section 2 with an investigation of the dual of a space with a flat spot.

Complementing these results, we identify in Section 3 two more properties of Banach spaces which rule out the existence of flat spots. One has to do with the existence of certain projections in the dual space and the other is the Kadec-Klee property of the norm. Joined with Pach's theorem, our results show the existence of 'bad' renormings for non-superreflexive spaces. We conclude with some remarks on open problems.

\section{Existence of flat spots in non-flat Banach spaces}

Proposition 1.1 (Pach (1979)). On every non-superreflexive Banach space $(X,\|\|$.$) there exists an equivalent norm \|\cdot\|$ such that $(X,\|\|$.$) has a flat spot.$

The proof of this result is rather complicated and will appear elsewhere. The simplest example of a space for which this renorming (to obtain a flat spot) can be carried out explicitly is $c_{0}=c_{0}(\mathbf{N})$. Observe that $c_{0}$ is not isomorphic to a flat space, since its dual is separable (Karlovitz (1973)). We now reproduce this example from van Dulst and Schäffer (1979). As usual, we denote the space $l^{\infty}(\Gamma)$ by $l_{n}^{\infty}$ if 
$\Gamma=\{1,2, \ldots, n\}(n \in \mathbf{N})$. Also, when more than one space is involved, we use its name as a subscript, for example $m_{X}(x), m_{Y}(x)$. Let us note the trivial inequality $m_{X}(x) \leqslant m_{Y}(x)$ for every $x \in S_{Y}$, where $Y$ is a subspace of $X$.

EXAmple. 1. Let $\Gamma$ be the set of all rational numbers in $[-1,1]$ and define $q \in l^{\infty}(\Gamma)$ by $q(\gamma):=\gamma$ for all $\gamma \in \Gamma$. We consider the subspace $X:=c_{0}(\Gamma)+\mathbf{R} q$ of $l^{\infty}(\Gamma)$. $X$ is obviously isomorphic to $c_{0}(\mathbf{N})$, since $\Gamma$ is countably infinite. Furthermore, $\|q\|=1$. We shall prove that $q$ is a flat spot of $X$.

2. Let $n \in \mathbf{N}$ be fixed for the time being. We define a map $T_{n}: l_{n+1}^{\infty} \rightarrow l^{\infty}(\Gamma)$ by

$$
\left(T_{n} x\right)(\gamma):= \begin{cases}x(j) & \text { if } \gamma=1-2(j-1) / n \quad(j=1, \ldots, n+1), \\ x(1) \gamma & \text { if } \gamma \in \Gamma \backslash\{1-2(j-1) / n: j=1, \ldots, n+1\}\end{cases}
$$

for $x \in l_{n+1}^{\infty}$. It is plain that $T_{n}$ is linear and isometric. We denote its range by $X_{n}$. Clearly $T_{n} x-x(1) q$ has finite support for every $x \in l_{n+1}^{\infty}$; therefore $X_{n}$ is a subspace of $X$. Define $p_{0} \in S_{l_{n+1}{ }^{\infty}}$ by

$$
p_{0}(j):=1-2(j-1) / n \quad(j=1, \ldots, n+1) .
$$

It follows that $\left(T_{n} p_{0}\right)(\gamma)=\gamma$ for all $\gamma \in \Gamma$, so that $T_{n} p_{0}=q$.

3. We claim that $m_{l_{n+1}^{\infty}}\left(p_{0}\right) \leqslant 2(1+1 / n)$. Indeed, consider the polygon in $l_{n+1}^{\infty}$ joining $p_{0}$ to $-p_{0}$ and with consecutive vertices

$$
\begin{aligned}
p_{0}: & =\left(1,1-\frac{2}{n}, 1-\frac{4}{n}, \ldots,-1+\frac{4}{n},-1+\frac{2}{n},-1\right) \\
p_{1}: & =\left(1,1,1-\frac{2}{n}, \ldots,-1+\frac{6}{n},-1+\frac{4}{n},-1+\frac{2}{n}\right) \\
p_{2}: & =\left(1-\frac{2}{n}, 1,1, \ldots,-1+\frac{8}{n},-1+\frac{6}{n},-1+\frac{4}{n}\right) \\
. & . \quad . \quad . . . . . . . . . \\
p_{n}: & =\left(-1+\frac{2}{n},-1+\frac{4}{n},-1+\frac{6}{n}, \ldots, 1-\frac{2}{n}, 1,1\right) \\
p_{n+1}: & =-p_{0}=\left(-1,-1+\frac{2}{n},-1+\frac{4}{n}, \ldots, 1-\frac{4}{n}, 1-\frac{2}{n}, 1\right) .
\end{aligned}
$$

Clearly $\left\|p_{i}\right\|=1(i=0, \ldots, n+1)$. Furthermore, since $p_{i-1}(i)=p_{i}(i)=1$ for all $i=1, \ldots, n+1$, each segment $\left[p_{i-1}, p_{i}\right]$, and therefore the entire polygon, lies in $S_{t_{n+1}^{\infty}}$. Obviously, $\left\|p_{i}-p_{i-1}\right\|=2 / n$ for all $i=1, \ldots, n+1$, so that the polygon has length $(n+1)(2 / n)=2(1+1 / n)$. This proves our claim. 
4. Finally, since $T_{n}$ is an isometry from $l_{n+1}^{\infty}$ onto $X_{n}$, we now conclude that

$$
m_{X}(q) \leqslant m_{X_{n}}(q)=m_{X_{n}}\left(T_{n} p_{0}\right)=m_{l_{n+1}}\left(p_{0}\right) \leqslant 2(1+1 / n) .
$$

Thus $2 \leqslant m_{X}(q) \leqslant 2(1+1 / n)$. Since $n \in \mathbb{N}$ was arbitrary, it follows that $m_{X}(q)=2$, so that $q$ is a flat spot of $X$.

\section{The geometry of the unit sphere at flat spots}

In this section, $X$ will denote a normed linear space. For any $x \in S_{X}$, we define the local moduli of convexity $\delta(x,$.$) and of smoothness \rho(x,$.$) by$

$$
\begin{array}{ll}
\delta(x, \tau)=\inf \left\{1-\frac{1}{2}\|x+y\|: y \in S_{X},\|x-y\| \geqslant \tau\right\} & (0 \leqslant \tau \leqslant 2), \\
\rho(x, \tau)=\sup \left\{\frac{1}{2}\|x+y\|+\frac{1}{2}\|x-y\|-1:\|y\|=\tau\right\} & (\tau \geqslant 0) .
\end{array}
$$

$X$ is called uniformly non-square if there exists a $\delta>0$ such that there do not exist $x, y \in S_{X}$ with

$$
\frac{1}{2}\|x+y\|>1-\delta \text { and } \frac{1}{2}\|x-y\|>1-\delta .
$$

$x \in S_{X}$ is called a strongly exposed point of $B_{X}$ if there exists a support functional $x^{*}$ at $x$, that is $\left\|x^{*}\right\|=\left\langle x, x^{*}\right\rangle=1$, such that $\left\langle x_{n}, x^{*}\right\rangle \rightarrow 1, x_{n} \in B_{X}(n=1,2, \ldots)$ implies $\left\|x_{n}-x\right\| \rightarrow 0$.

We begin now by deriving some inequalities of which the results in this section will be easy consequences. Let $x \in S_{X}$ and let a curve be given in $S_{X}$ with initial point $x$ and endpoint $-x$ and with length $2+\varepsilon$, where $\varepsilon>0$. Suppose that $x_{\varepsilon}:[0,2+\varepsilon] \rightarrow S_{X}$ is its standard representation in terms of arc length. Then

$$
x_{\varepsilon}(0)=x=-x_{\varepsilon}(2+\varepsilon)
$$

and

$$
\left\|x_{\varepsilon}(s+h)-x_{\varepsilon}(s)\right\| \leqslant h,
$$

whenever $0 \leqslant s \leqslant s+h \leqslant 2+\varepsilon$. Let $x_{\varepsilon}^{*}:[0,2+\varepsilon] \rightarrow S_{X}$, be such that

$$
\left\langle x_{\varepsilon}(t), x_{s}^{*}(t)\right\rangle=1 \text { for all } 0 \leqslant t \leqslant 2+\varepsilon
$$

In other words, $x_{\varepsilon}^{*}(t)$ is an arbitrary support functional at $x_{\varepsilon}(t), 0 \leqslant t \leqslant 2+\varepsilon$.

We claim that, for any $0 \leqslant t, s \leqslant 2+\varepsilon$,

$$
1-|s-t| \leqslant\left\langle x_{\varepsilon}(s), x_{\varepsilon}^{*}(t)\right\rangle \leqslant 1-|s-t|+\varepsilon .
$$

Indeed, by (2) and (3),

$$
\begin{aligned}
\left|\left\langle x_{\varepsilon}(s), x_{\varepsilon}^{*}(t)\right\rangle-1\right| & =\left|\left\langle x_{\varepsilon}(s), x_{\varepsilon}^{*}(t)\right\rangle-\left\langle x_{\varepsilon}(t), x_{\varepsilon}^{*}(t)\right\rangle\right| \\
& \leqslant\left\|x_{\varepsilon}(s)-x_{\varepsilon}(t)\right\| \leqslant|s-t|,
\end{aligned}
$$


and, using also (1),

(6)

$$
\begin{aligned}
\left\langle x_{\varepsilon}(s), x_{\varepsilon}^{*}(t)\right\rangle+1 & =\left\langle x_{\varepsilon}(s), x_{\varepsilon}^{*}(t)\right\rangle+\left\langle x_{\varepsilon}(t), x_{\varepsilon}^{*}(t)\right\rangle \\
& \leqslant\left\|x_{\varepsilon}(s)+x_{\varepsilon}(t)\right\| \leqslant\left\{\begin{array}{c}
\left\|x_{\varepsilon}(s)-x_{\varepsilon}(0)\right\| \\
+\| x_{\varepsilon}(2+\varepsilon)-x_{\varepsilon}(t) \mid \leqslant s+(2+\varepsilon-t) \\
\left\|x_{\varepsilon}(s)-x_{\varepsilon}(2+\varepsilon)\right\| \\
+\left\|x_{\varepsilon}(0)-x_{\varepsilon}(t)\right\| \leqslant(2+\varepsilon-s)+t .
\end{array}\right.
\end{aligned}
$$

From (5) and (6), (4) easily follows.

Next, for any $0<t \leqslant 2+\varepsilon$, let us define

$$
x_{s, t}=\frac{x_{8}(t)-x_{8}(0)}{\left\|x_{8}(t)-x_{8}(0)\right\|} .
$$

Then $\left\|x_{\varepsilon, d}\right\|=1$ and by (3), (4) and (2) we have

$$
\begin{aligned}
\left\langle x_{\epsilon,}, x_{\varepsilon}^{*}(t)\right\rangle & =\frac{\left\langle x_{\varepsilon}(t), x_{\varepsilon}^{*}(t)\right\rangle-\left\langle x_{\varepsilon}(0), x_{\varepsilon}^{*}(t)\right\rangle}{\left\|x_{\varepsilon}(t)-x_{\epsilon}(0)\right\|}=\frac{1-\left\langle x_{\varepsilon}(0), x_{\varepsilon}^{*}(t)\right\rangle}{\left\|x_{\varepsilon}(t)-x_{\varepsilon}(0)\right\|} \\
& \geqslant \frac{1-(1-t+\varepsilon)}{t}=\frac{t-\varepsilon}{t},
\end{aligned}
$$

and

$$
\begin{aligned}
\left\langle x_{\varepsilon, t}, x_{\varepsilon}^{*}(0)\right\rangle & =\frac{\left\langle x_{\varepsilon}(t), x_{\varepsilon}^{*}(0)\right\rangle-\left\langle x_{\varepsilon}(0), x_{\varepsilon}^{*}(0)\right\rangle}{\left\|x_{\varepsilon}(t)-x_{\varepsilon}(0)\right\|}=\frac{\left\langle x_{\varepsilon}(t), x_{\varepsilon}^{*}(0)\right\rangle-1}{\left\|x_{\varepsilon}(t)-x_{\varepsilon}(0)\right\|} \\
& \leqslant \frac{(1-t+\varepsilon)-1}{t}=\frac{-t+\varepsilon}{t} .
\end{aligned}
$$

From these inequalities we derive

LEMMA 2.1. Let $x \in S_{X}, \varepsilon>0$, and suppose that $x_{\varepsilon}:[0,2+\varepsilon] \rightarrow S_{X}$ is the standard representation in terms of arc length of a curve with initial point $x$ and endpoint $-x$. Then, with $x_{e, t}$ defined as above, we have

$$
\text { (i) } \frac{\left\|x+x_{\epsilon,(2 \varepsilon)+}\right\|}{2}+\frac{\left\|x-x_{\epsilon,(2 \varepsilon)\}}\right\|}{2} \geqslant 2-(2 \varepsilon)^{\frac{1}{1}} \text {, }
$$

(ii) $\left\langle-x_{\varepsilon,(2 \varepsilon)\}}, x^{*}\right\rangle \geqslant 1-\left(\frac{1}{2} \varepsilon\right)^{\frac{1}{2}}$ for all support functionals $x^{*}$ at $x$,

(iii) $\rho(x, \tau) \geqslant \tau-(2 \tau \varepsilon)^{1}$ for all $\tau \geqslant 0$,

(iv) $\delta(x, \tau) \leqslant \varepsilon^{1}$ for all $0 \leqslant \tau \leqslant 2-\varepsilon^{1}$.

Proof. By (1), (3) and (8), we have, whenever $\tau \geqslant 0$ and $0<t \leqslant 2+\varepsilon$,

(9)

$$
\begin{aligned}
\left\|x-\tau x_{\epsilon, t}\right\| & =\left\|x_{\varepsilon}(0)-\tau x_{\varepsilon, t}\right\| \geqslant\left\langle x_{\varepsilon}(0)-\tau x_{\epsilon, t}, x_{\varepsilon}^{*}(0)\right\rangle \\
& =1-\tau\left\langle x_{\varepsilon, t}, x_{\varepsilon}^{*}(0)\right\rangle \geqslant 1-\tau\left(\frac{-t+\varepsilon}{t}\right)=1+\tau-\frac{\tau \varepsilon}{t},
\end{aligned}
$$


and, by (1), (4) and (7),

$$
\begin{aligned}
\left\|x+\tau x_{\epsilon, i}\right\| & =\left\|x_{\varepsilon}(0)+\tau x_{\varepsilon, \lambda}\right\| \geqslant\left\langle x_{\varepsilon}(0), x_{\varepsilon}^{*}(t)\right\rangle+\tau\left\langle x_{\varepsilon, i}, x_{\varepsilon}^{*}(t)\right\rangle \\
& \geqslant(1-t)+\tau\left(\frac{t-\varepsilon}{t}\right)=1+\tau-t-\frac{\tau \varepsilon}{t} .
\end{aligned}
$$

Substituting $\tau=1$ and $t=(2 \varepsilon)^{1}$ in (9) and (10) easily yields (i). More generally, (9) and (10) imply that, whenever $\tau \geqslant 0$ and $0<t \leqslant 2+\varepsilon$,

$$
\rho(x, \tau) \geqslant \frac{1}{2}\left\|x+\tau x_{\varepsilon, t}\right\|+\frac{1}{2}\left\|x-\tau x_{\varepsilon, t}\right\|-1 \geqslant \tau-\frac{1}{2} t-\frac{\tau \varepsilon}{t} .
$$

Hence, for all $\tau \geqslant 0$,

$$
\rho(x, \tau) \geqslant \tau-\inf _{0<1 \leqslant 2+\varepsilon}\left(\frac{\tau \varepsilon}{t}+\frac{1}{2} t\right)=\tau-(2 \tau \varepsilon)^{t},
$$

which is (iii). Next, by (8) we have, for all $0<t \leqslant 2+\varepsilon,\left\langle-x_{\varepsilon, t}, x_{\varepsilon}^{*}(0)\right\rangle \geqslant(t-\varepsilon) / t$. Since $x_{e}^{*}(0)$ is an arbitrary support functional at $x=x_{\varepsilon}(0)$, substitution of $t=(2 \varepsilon)^{*}$ gives (ii). Finally, to prove (iv), note that, by (9), $\left\|x-x_{\varepsilon, \varepsilon}\right\| \geqslant 2-\varepsilon^{\ddagger}$, and, by (10), $\left\|x+x_{\varepsilon, \epsilon \varepsilon}\right\| \geqslant 2-2 \varepsilon^{\frac{1}{3}}$; so $\delta\left(x, 2-\varepsilon^{i}\right) \leqslant 1-\frac{1}{2}\left\|x+x_{\varepsilon, \epsilon}\right\| \leqslant 1-\frac{1}{2}\left(2-2 \varepsilon^{i}\right)=\varepsilon^{\frac{1}{4}}$. From this (iv) follows, since $\delta(x,$.$) is a non-decreasing function.$

REMARK 2.2. (i) means that the unit sphere of the two-dimensional subspace spnaned by the unit vectors $x$ and $x_{\varepsilon,(2 \varepsilon)\}}$ resembles a square with vertices $\pm x$, $\pm x_{\varepsilon,(2 \varepsilon) t}$ if $\varepsilon$ is small. (Compare Schäffer (1976), Theorem $17 \mathrm{H}$ for a similar result.) (ii) means that, moreover, the edge joining $x$ and $-x_{\varepsilon,(2 \varepsilon) 4}$ lies uniformly close to all support hyperplanes at $x$, if $\varepsilon$ is small.

Proposition 2.3. If $x$ is a flat spot in $X$, then

(i) for every $\eta>0$ there exists a $y \in S_{X}$ such that

$$
\frac{1}{2}\|x+y\|+\frac{1}{2}\|x-y\| \geqslant 2-\eta,
$$

and such that, in addition,

(ii) $\left\langle y, x^{*}\right\rangle \geqslant 1-\eta$ for all support functionals $x^{*}$ at $x$,

(iii) $\rho(x, \tau)=\tau$ for all $\tau \geqslant 0$,

(iv) $\delta(x, \tau)=0$ for all $0 \leqslant \tau<2$.

In particular, the norm is not Fréchet differentiable at $x$, is not locally uniformly convex at $x$, and $x$ fails to be a strongly exposed point of $B_{X}$.

ProOF. (i) and (ii) immediately follow from the corresponding properties in Lemma 2.1, by taking $y=x_{\varepsilon,(2 \varepsilon) t}$ for sufficiently small $\varepsilon>0$. Moreover, taking the limit for $\varepsilon \rightarrow 0$ in (iii) and (iv) of Lemma 2.1, we get $\rho(x, \tau) \geqslant \tau(\tau \geqslant 0)$ and $\delta(x, \tau) \leqslant 0(0 \leqslant \tau<2)$. Since the reverse inequalities always hold, this proves (iii) 
and (iv). As for the remaining statements, recall that $\|$.$\| is Fréchet differentiable$ at $x$ if and only if $\lim _{\tau \rightarrow 0} \rho(x, \tau) / \tau=0$. Finally, the fact that $x$ is not strongly exposed is obvious, since, by (i) and (ii), for any support functional $x^{*}$ at $x$ and any $\eta>0$, the set $\left\{y \in S_{X}:\left\langle y, x^{*}\right\rangle \geqslant 1-\eta\right\}$ has diameter 2 .

Remark 2.4. (a) It follows in particular from Lemma 2.1(i), by taking $\varepsilon$ arbitrarily small, that if $m(X)=2, X$ fails to be uniformly non-square and $a$ fortiori uniformly convex. It is known that $X$ is not even isomorphic to a uniformly non-square space if $m(X)=2$. This deep result is due to Schäffer, James and Enflo (see James and Schäffer (1972), Enflo (1972), James (1972)). The pleasant feature in the present discussion is that, even if $m(X)$ is greater than but close to 2, we are able to locate 'squares' precisely, working with curves whose lengths approximate the girth of the unit ball. And also, of course, if the girth is achieved 'locally', that is if $x \in S_{X}$ is a flat spot, then 'good' squares can be found with vertex in $x$ and 'almost' tangent to all support hyperplanes.

Observe also that Proposition 2.3 never holds with $\eta=0$. Indeed, if

$$
\|x+y\|=\|x-y\|=1
$$

for some $y \in S_{X}$, then the subspace spanned by $x$ and $y$ has norm

$$
\|\alpha x+\beta y\|=|\alpha|+|\beta|,
$$

and so there exists a support functional $x^{*}$ at $x$ with $\left\langle y, x^{*}\right\rangle=0$, contradicting (ii).

(b) If $X^{*}$ is WCG, then $X$ is an SDS (Namioka and Phelps (1975)). Therefore, by Proposition 2.3, $\left\{x \in S_{X}: m(x)=2\right\}$ is nowhere dense in $S_{X}$. For an atomless measure $\mu$ the space $X=L^{1}(\mu)$ has the property that $m(x)=2$ for all $x \in S_{X}$ (Schäffer (1971)). This shows that a flat spot may very well be smooth. In fact, if $L^{1}(\mu)$ is separable, it is a WDS (Asplund (1968)). Let us finally observe (Schäffer (1976)) that every WCG space has an equivalent norm for which no flat spots exist. This is a consequence of Proposition 2.3 since every WCG space has an equivalent locally uniformly convex norm (Troyanski (1971)).

Next we show that if $m(x)=2$ for some $x \in S_{X}$, then the geometric situation at $x$ described in Proposition 2.3 also largely holds in $X^{*}$ at any support functional $x^{*}$ at $x$.

Proposition 2.5. Let $x$ be a flat spot in $X$. Then

(i) for every $\eta>0$ there exists a $y^{*} \in S_{X^{*}}$ such that

$$
\frac{1}{2}\left\|x^{*}+y^{*}\right\|+\frac{1}{2}\left\|x^{*}-y^{*}\right\| \geqslant 2-\eta,
$$

for all support functionals $x^{*}$ at $x$ and such that, in addition,

(ii) $\left\langle x, y^{*}\right\rangle \geqslant 1-\eta$,

(iii) $\rho\left(x^{*}, \tau\right)=\tau$ for all $\tau \geqslant 0$, and

(iv) $\delta\left(x^{*}, \tau\right)=0$ for all $0 \leqslant \tau<2$, for all support functionals $x^{*}$ at $x$. 
Proof. By Proposition 2.3 there exists for any $\eta_{1}>0$ a $y \in S_{X}$ such that $\frac{1}{2}\|x+y\|+\frac{1}{2}\|x-y\| \geqslant 2-\eta_{1}$ and $\left\langle-y, x^{*}\right\rangle \geqslant 1-\eta_{1}$ for all support functionals $x^{*}$ at $x$.

Clearly the first inequality implies $\|x+y\| \geqslant 2-2 \eta_{1}$. Let $y^{*} \in S_{X^{*}}$ be a support functional at $(x+y) /\|x+y\|$, that is $\left\langle x+y, y^{*}\right\rangle=\|x+y\|$. Then, for any $\tau \geqslant 0$, and any support functional $x^{*}$ at $x$,

$$
\begin{aligned}
\left\|x^{*}+\tau y^{*}\right\|+\left\|x^{*}-\tau y^{*}\right\| & \geqslant\left\langle x, x^{*}+\tau y^{*}\right\rangle+\left\langle-y, x^{*}-\tau y^{*}\right\rangle \\
& =\left\langle x, x^{*}\right\rangle+\left\langle-y, x^{*}\right\rangle+\tau\left\langle x+y, y^{*}\right\rangle \\
& \geqslant 1+\left(1-\eta_{1}\right)+\tau\|x+y\| \\
& \geqslant 1+\left(1-\eta_{1}\right)+\tau\left(2-2 \eta_{1}\right) \\
& =2+2 \tau-\eta_{1}(1+2 \tau) .
\end{aligned}
$$

Moreover,

$$
\left\langle x, y^{*}\right\rangle=\|x+y\|-\left\langle y, y^{*}\right\rangle \geqslant\left(2-2 \eta_{1}\right)-1=1-2 \eta_{1} .
$$

Since (11) with $\tau=1$ easily yields $\left\|x^{*} \pm y^{*}\right\| \geqslant 2-3 \eta_{1}$, we have

$$
\delta\left(x^{*}, 2-3 \eta_{1}\right) \leqslant 1-\frac{1}{2}\left\|x^{*}+y^{*}\right\| \leqslant 1-\frac{1}{2}\left(2-3 \eta_{1}\right)=\frac{3}{2} \eta_{1} .
$$

(i) now follows from (11) with $\tau=1$ and $\eta_{1}$ sufficiently small, and similarly (ii) follows from (12). (iii) and (iv) are obtained from (11) and (13) by letting $\eta_{1}$ tend to 0 .

Remark 2.6. Observe that, in contrast to Proposition 2.3, the squares with vertices $\pm x^{*}, \pm y^{*}$ in this proof have an edge close to the support hyperplane at $x^{*}$ defined by $x$, but not necessarily to all support hyperplanes at $x^{*}$. Also note that $x^{*}$ need not be a flat spot in $X^{*}$. Indeed, if $x$ is the function identically 1 in $X=L^{1}[0,1]$ and if $x^{*}$ is the same function in $X^{*}=L^{\infty}[0,1]$, then

$$
\left\langle x, x^{*}\right\rangle=\|x\|=\left\|x^{*}\right\|=1, \quad m(x)=2 \text { and } m\left(x^{*}\right)=4,
$$

as is easily verified (Schäffer (1976)). It can in fact be shown (van Dulst and Schäffer (1979)) that, in contrast to the situation for flat spaces, there exists a space with a flat spot whose dual is separable and has no flat spots.

In conjunction with Proposition 1.1 the results obtained so far in this section show that even for reflexive $X$ the geometry of $S_{X}$ can be quite pathological. 
Proposition 2.7. Any non-superreflexive Banach space $X$ for which $B_{X^{*}}$ is $w^{*}$-sequentially compact can be equivalently renormed so that there exist elements $x_{n}^{*} \in S_{X^{*}}(n=1,2, \ldots), x_{0}^{*} \in S_{X^{*}}$ satisfying the following conditions:

$$
\begin{gathered}
w^{*}-\lim _{n \rightarrow \infty} x_{n}^{*}=x_{0}^{*}, \\
\lim _{n \rightarrow \infty}\left\|x_{n}^{*}-x_{0}^{*}\right\|=2, \\
\lim _{n, m \rightarrow \infty ; n \neq m}\left\|x_{n}^{*}-x_{m}^{*}\right\|=2 .
\end{gathered}
$$

Proof. Using Proposition 1.1 choose an equivalent norm so that $X$ has a flat spot $x$. For each $n \in \mathbf{N}$, pick $\varepsilon_{n}>0$ such that $\left(2 \varepsilon_{n}\right)^{4}<n^{-1}$. With the notations used above, put $x_{n}=x_{\left.\varepsilon_{n},\left(2 \varepsilon_{n}\right)\right\}}(n=1,2, \ldots)$. Then, by Lemma 2.1,

$$
\frac{1}{2}\left\|x+x_{n}\right\|+\frac{1}{2}\left\|x-x_{n}\right\| \geqslant 2-n^{-1}
$$

and for all $x^{*} \in S_{X^{*}}$ satisfying $\left\langle x, x^{*}\right\rangle=1$ we have

$$
\left\langle-x_{n}, x^{*}\right\rangle>1-2 n^{-1} \text {. }
$$

Now choose $x_{n}^{*} \in S_{X^{*}}$ such that $\left\langle x+x_{n}, x_{n}^{*}\right\rangle=\left\|x+x_{n}\right\|$. The proof of Proposition 2.5 then shows that for all $x^{*} \in S_{X^{*}}$ with $\left\langle x, x^{*}\right\rangle=1$ we have

$$
\left\|x^{*}-x_{n}^{*}\right\| \geqslant 2-3 n^{-1}
$$

and

$$
\left\langle x, x_{n}^{*}\right\rangle \geqslant 1-2 n^{-1} .
$$

By passing to a subsequence we may assume that $\left\{x_{n}^{*}\right\}$ is $w^{*}$-convergent, with limit $x_{0}^{*}$, say. By (17), $\left\langle x, x_{0}^{*}\right\rangle=1$ and so, by (16), $\left\|x_{0}^{*}-x_{n}^{*}\right\| \geqslant 2-3 n^{-1}(n=1,2, \ldots)$. Thus (i) and (ii) hold. It is easily verified that by passing to yet another subsequence and using (i) and (ii), also (iii) can be realized.

\section{Some Banach spaces having no flat spots}

The next result is most easily stated in terms of a parameter (see Karlovitz (1976)) which we now introduce. If $X$ is a Banach space and $x^{*} \in S_{X^{*}}$, we define $\lambda\left(x^{*}\right)$ to be the infimum of the positive numbers $\alpha$ for which there exists a $w^{*}$-closed subspace $M \subset X^{*}$ with codim $M<\infty$ such that the projection from $\operatorname{sp} x^{*} \oplus M$ onto $M$ with kernel sp $x^{*}$ has norm $\leqslant \alpha$. In formula,

$$
\begin{aligned}
\lambda\left(x^{*}\right)=\inf \{\alpha>0: & \exists M \subset X^{*}, M w^{*} \text {-closed, } \operatorname{codim} M<\infty, \text { and } \\
& \left.\left\|y^{*}+\beta x^{*}\right\| \geqslant \alpha^{-1}\left\|y^{*}\right\| \text { for all } y^{*} \in M \text { and all scalars } \beta\right\} .
\end{aligned}
$$

We also set $\lambda\left(X^{*}\right)=\sup \left\{\lambda\left(x^{*}\right): x^{*} \in S_{X^{*}}\right\}$. 
Observe that $\lambda\left(X^{*}\right) \leqslant 2$ for all $X$. Indeed, let $x_{0}^{*} \in S_{X^{*}}$ and $\varepsilon>0$ be arbitrary. By subreflexivity (Bishop and Phelps (1961)), there exists a $y_{0}^{*} \in S_{X^{*}}$ and a $y_{0} \in S_{X}$ such that $\left\langle y_{0}, y_{0}^{*}\right\rangle=1$ and $\left\|y_{0}^{*}-x_{0}^{*}\right\| \leqslant \varepsilon$. Put $M=\left(\operatorname{sp} y_{0}\right)^{\perp}$. Fix $y^{*} \in S_{M}$. Then we have, whenever $|\beta| \geqslant \frac{1}{2}$,

$$
\begin{aligned}
\left\|y^{*}+\beta x_{0}^{*}\right\| & \geqslant\left\|y^{*}+\beta y_{0}^{*}\right\|-|\beta|\left\|x_{0}^{*}-y_{0}^{*}\right\| \geqslant\left|\left\langle y_{0}, y^{*}+\beta y_{0}^{*}\right\rangle\right|-|\beta| \varepsilon \\
& =|\beta|-|\beta| \varepsilon \geqslant \frac{1}{2}(1-\varepsilon),
\end{aligned}
$$

and, whenever $|\beta| \leqslant \frac{1}{2}$,

$$
\left\|y^{*}+\beta x_{0}^{*}\right\| \geqslant\left\|y^{*}\right\|-|\beta|=1-|\beta| \geqslant \frac{1}{2} .
$$

Thus $\lambda\left(x_{0}^{*}\right) \leqslant 2$, since $\varepsilon>0$ was arbitrary.

Proposition 3.1. Let $X$ be a normed linear space with a flat spot $x_{0}$. Then $\lambda\left(x_{0}^{*}\right)=2$ for some support functional $x_{0}^{*}$ at $x_{0}$. Hence $\lambda\left(X^{*}\right)<2$ implies that $X$ has no flat spots.

Proof. By Proposition 2.3 there exists a sequence $\left\{x_{n}\right\} \subset S_{X}$ satisfying

$$
\frac{1}{2}\left\|x_{0}+x_{n}\right\|+\frac{1}{2}\left\|x_{0}-x_{n}\right\| \geqslant 2-n^{-1} \quad(n=1,2, \ldots)
$$

and

$$
\left\langle-x_{n}, x^{*}\right\rangle \geqslant 1-n^{-1} \text { for all support functionals } x^{*} \text { at } x_{0} \quad(n=1,2, \ldots) .
$$

Let $x_{n}^{*} \in S_{X}$ be such that

$$
\left\langle x_{0}+x_{n}, x_{n}^{*}\right\rangle=\left\|x_{0}+x_{n}\right\| \quad(n=1,2, \ldots) .
$$

The proof of Proposition 2.5 then shows that for all support functionals $x^{*}$ at $x_{0}$ we have

$$
\frac{1}{2}\left\|x^{*}+x_{n}^{*}\right\|+\frac{1}{2}\left\|x^{*}-x_{n}^{*}\right\| \geqslant 2-3 n^{-1} \quad(n=1,2, \ldots),
$$

as well as

$$
\left\langle x_{0}, x_{n}^{*}\right\rangle \geqslant 1-2 n^{-1} \quad(n=1,2, \ldots) .
$$

Let $x_{0}^{*} \in B_{X^{*}}$ be a $w^{*}$ limit point of $\left\{x_{n}^{*}\right\}$. Clearly, by $(21),\left\langle x_{0}, x_{0}^{*}\right\rangle=1$; so $x_{0}^{*}$ is a support functional at $x_{0}$. In particular, by (20),

$$
\frac{1}{2}\left\|x_{0}^{*}+x_{n}^{*}\right\|-\frac{1}{2}\left\|x_{0}^{*} \pm x_{n}^{*}\right\| \geqslant 2-3 n^{-1} \quad(n=1,2, \ldots) .
$$

We claim that $\lambda\left(x_{0}^{*}\right)=2$. Indeed, assume for contradiction that $\lambda\left(x_{0}^{*}\right)<2$. Then there exists a $w^{*}$-closed subspace $M \subset X^{*}$ with $\operatorname{codim} M<\infty$ such that the projection $P$ from sp $x_{0}^{*} \oplus M$ onto $M$ with kernel sp $x_{0}^{*}$ has norm $\|P\|=2-\varepsilon<2$. 
Choose a finite biorthogonal system $y_{1}, \ldots, y_{n} \in X, y_{1}^{*}, \ldots, y_{n}^{*} \in X^{*}$ such that

$$
M=\left(\operatorname{sp}\left\{y_{1}, \ldots, y_{n}\right\}\right)^{\perp} .
$$

Then any $x^{*} \in X^{*}$ can be uniquely written as

$$
x^{*}=\sum_{i=1}^{n}\left\langle y_{i}, x^{*}\right\rangle y_{i}^{*}+z^{*}, \quad \text { with } z^{*} \in M .
$$

Now consider the sequence $\left\{\frac{1}{2}\left(x_{0}^{*}-x_{n}^{*}\right)\right\}$. By (22), $\lim _{n \rightarrow \infty}\left\|\frac{1}{2}\left(x_{0}^{*}-x_{n}^{*}\right)\right\|=1$. Since also 0 is a $w^{*}$-limit point, it follows from (23) that there exists an $n \in \mathbf{N}$ and a $z_{n}^{*} \in M$ such that

$$
\left\|z_{n}^{*}\right\|=1 \text { and }\left\|z_{n}^{*}-\frac{1}{2}\left(x_{0}^{*}-x_{n}^{*}\right)\right\|<\frac{1}{4} \varepsilon .
$$

From $\|P\|=2-\varepsilon$ and $z_{n}^{*} \in M$ we deduce the existence of an $x_{n}^{* *} \in X^{* *}$ satisfying

$$
\left\langle z_{n}^{*}, x_{n}^{* *}\right\rangle=1,\left\langle x_{0}^{*}, x_{n}^{* *}\right\rangle=0 \text { and }\left\|x_{n}^{* *}\right\| \leqslant 2-\varepsilon .
$$

In particular, by (24),

$$
\begin{aligned}
\frac{1}{2}\left|\left\langle x_{n}^{*}, x_{n}^{* *}\right\rangle\right| & =\left|\left\langle\frac{1}{2}\left(x_{0}^{*}-x_{n}^{*}\right), x_{n}^{* *}\right\rangle\right| \geqslant\left\langle z_{n}^{*}, x_{n}^{* *}\right\rangle-\left\|z_{n}^{*}-\frac{1}{2}\left(x_{0}^{*}-x_{n}^{*}\right)\right\|\left\|x_{n}^{* *}\right\| \\
& \geqslant 1-\frac{1}{4} \varepsilon(2-\varepsilon)>1-\frac{1}{2} \varepsilon .
\end{aligned}
$$

Hence $\left|\left\langle x_{n}^{*}, x_{n}^{* *}\right\rangle\right|>2-\varepsilon$, contradicting $\left\|x_{n}^{* *}\right\| \leqslant 2-\varepsilon$. This completes the proof.

COROLLARY 3.2. Let $X$ be a Banach space with a shrinking finite-dimensional Schauder decomposition (FDD) $\left\{X_{n}\right\}$, with associated sequence of projections $\left\{P_{n}\right\}\left(P_{n}\left(\sum_{i=1}^{\infty} x_{i}\right)=\sum_{i=1}^{n} x_{i}, x_{i} \in X_{i}\right)$. If $\liminf _{n \rightarrow \infty}\left\|I-P_{n}\right\|<2$, then $X$ has no flat spots. In particular, $X$ has no flat spots if it has an unconditional shrinking $F D D$ with unconditional decomposition constant $<2$.

Proor. The last statement is an obvious consequence of the first. To prove the first, let $x^{*} \in S_{X^{*}}$ be arbitrary. By passing to a subsequence if necessary, we may suppose that $\left\|I-P_{n}\right\| \leqslant 2-\varepsilon$ for all $n \in \mathbf{N}$ and some $\varepsilon>0$. Since $\left\{X_{n}\right\}$ is shrinking, $\lim _{n \rightarrow \infty}\left\|x^{*}-P_{n}^{*} x^{*}\right\|=0$. Combining this with $\left\|I-P_{n}^{*}\right\| \leqslant 2-\varepsilon(n=1,2, \ldots)$, we readily deduce that for sufficiently large $n$ the projection from $\operatorname{sp} x^{*} \oplus\left(I-P_{n}^{*}\right) X^{*}$ onto the $w^{*}$-closed finite-codimensional subspace $\left(I-P_{n}^{*}\right) X^{*}$ with kernel $\operatorname{sp} x^{*}$ has norm $\leqslant 2-\frac{1}{2} \varepsilon$. Thus $\lambda\left(X^{*}\right) \leqslant 2-\frac{1}{2} \varepsilon$, and the conclusion follows from Proposition 3.1.

REMARK 3.3. (a) An interesting particular case is the Tsirelson space $T$ (Tsirelson (1974), Figiel and Johnson (1974)). It is reflexive, but contains no infinitedimensional superreflexive subspaces, that is $m(Y)=2$ for all infinite-dimensional 
$Y \subset T$. Nevertheless $T$ has no flat spots by Corollary 3.2, since it has an unconditionally monotone basis.

(b) Arguments similar to the ones used to prove Proposition 3.1 will show that if some $x^{*} \in S_{X^{*}}$ attaining its norm is a flat spot in $X^{*}$, then either $B_{X^{*}}$ is not strictly convex at $x^{*}$ or $\lambda\left(x^{*}\right)=2$. We do not give details.

In conjunction with Proposition 1.1, Corollary 3.2 yields the following renorming result, which seems to be new even for spaces with bases.

PROPOSITION 3.4. Let $X$ be a non-superreflexive Banach space with a shrinking $F D D$. Then there exists on $X$ an equivalent norm such that, with respect to this new norm, liminf ${ }_{n \rightarrow \infty}\left\|I-P_{n}\right\| \geqslant 2$ for every shrinking $F D D$ with associated sequence of projections $\left\{\boldsymbol{P}_{n}\right\}$.

Our final result exhibits yet another property which, under the mild assumption that $X$ is WCG, rules our the existence of flat spots in both $X$ and $X^{*}$. A norm on a Banach space $X$ is called a Kadec-Klee norm (KK norm) if on $S_{X^{*}} w^{*}$-sequential convergence implies norm-convergence, or, more precisely, if $x_{n}^{*} \stackrel{\omega^{*}}{\longrightarrow} x^{*}$ and $\left\|x_{n}^{*}\right\| \rightarrow\left\|x^{*}\right\|$ imply $\left\|x_{n}^{*}-x^{*}\right\| \rightarrow 0$. It is well known that every Banach space with a separable dual has an equivalent KK norm (Kadec (1952), Klee (1960/61)). The same result holds if $X$ and $X^{*}$ are both WCG since by John and Zizler (1972), $X$ then has an equivalent norm whose dual norm is locally uniformly convex. Such a norm is obviously KK. Note, however, that in general a KK norm and its dual do not have even the weakest smoothness and rotundity properties. (Consider, for example, the natural norms on $c_{0}$ and its dual $l^{\mathbf{1}}$.)

Proposimion 3.5. Let $X$ be a Banach space with a $K K$ norm and suppose that $B_{X} *$ is $w^{*}$-sequentially compact. Then the following hold.

(i) $X$ has no flat spots.

(ii) If $X^{*}$ has a flat spot, then $X^{*}$ is fat.

(iii) If $X$ is WCG, then $X^{*}$ fails to be flat; so $X^{*}$ has no flat spots.

Thus, if $X$ is a WCG space with a KK norm, then neither $X$ nor $X^{*}$ has flat spots.

Proof. The last statement follows from (i), (ii) and (iii) if one recalls the fact that for a WCG space $X B_{X^{*}}$ is an Eberlein compact in its $w^{*}$-topology (Amir and Lindenstrauss (1968), Lindenstrauss (1972)) and therefore $w^{*}$-sequentially compact.

(i) Suppose $x$ is a flat spot in $X$. The proof of Proposition 3.1 then shows that there exists a support functional $x^{*}$ at $x$ and a sequence $\left\{x_{n}^{*}\right\} \subset S_{X^{*}}$ such that $x_{n}^{*} \stackrel{w^{*}}{\longrightarrow} x^{*}$ and $\left\|x_{n}^{*}-x^{*}\right\| \rightarrow 2$. This contradicts the KK property.

(ii) Suppose $x^{*}$ is a flat spot in $X^{*}$. We show that then $X^{*}$ must be flat. For each $n \in \mathbf{N}$, let $\gamma_{n}$ be a curve in $S_{X^{*}}$ joining $x^{*}$ to $-x^{*}$ and with length $l\left(\gamma_{n}\right)<2+n^{-1}$. 
Let $x^{* *} \in X^{* *}$ be any support functional at $x^{*}$. Furthermore, let $A$ be a dense countable subset of the interval $(-1,1)$. For each $n \in \mathbf{N}$ and $\alpha \in A$ we choose a point $x_{n, \alpha}^{*} \in \gamma_{n} \cap\left\{y^{*}:\left\langle y^{*}, x^{* *}\right\rangle=\alpha\right\}$. Since $B_{X^{*}}$ is $w^{*}$-sequentially compact we may, by applying a diagonal procedure, assume that for each $\alpha \in A$ the sequence $\left\{x_{n, \alpha}^{*}\right\}_{n=1}^{\infty} w^{*}$-converges, say to $x_{\alpha}^{*}$.

We claim that $\lim _{n \rightarrow \infty}\left\|x_{\alpha}^{*}-x_{n, \alpha}^{*}\right\|=0$, for each $\alpha \in A$. Indeed, the inequalities

$$
\begin{aligned}
& \left\|x^{*}-x_{\alpha}^{*}\right\| \leqslant \liminf _{n \rightarrow \infty}\left\|x^{*}-x_{n, \alpha}^{*}\right\|, \\
& \left\|x^{*}+x_{\alpha}^{*}\right\| \leqslant \liminf _{n \rightarrow \infty}\left\|x^{*}+x_{n, \alpha}^{*}\right\|,
\end{aligned}
$$

which follow from the $w^{*}$-lower semicontinuity of the dual norm, together with

$$
2 \leqslant\left\|x^{*}-x_{n, \alpha}^{*}\right\|+\left\|x^{*}+x_{n, \alpha}^{*}\right\| \leqslant l\left(\gamma_{n}\right)<2+n^{-1}
$$

and

$$
2 \leqslant\left\|x^{*}-x_{\alpha}^{*}\right\|+\left\|x^{*}+x_{\alpha}^{*}\right\|,
$$

imply that, for all $\alpha \in A$,

$$
\left\|x^{*} \pm x_{\alpha}^{*}\right\|=\lim _{n \rightarrow \infty}\left\|x^{*} \pm x_{n, \alpha}^{*}\right\| \text { and }\left\|x^{*}-x_{\alpha}^{*}\right\|+\left\|x^{*}+x_{\alpha}^{*}\right\|=2 .
$$

Also, for all $n \in \mathbf{N}$ and $\alpha \in A$,

$$
\left\|x^{*}-x_{n, \alpha}^{*}\right\| \geqslant\left\langle x^{*}-x_{n, \alpha}^{*}, x^{* *}\right\rangle=1-\alpha
$$

and

$$
\left\|x^{*}+x_{n, \alpha}^{*}\right\| \geqslant\left\langle x^{*}+x_{n, \alpha}^{*}, x^{* *}\right\rangle=1+\alpha .
$$

Using (29) we conclude that, for all $\alpha \in A$,

$$
\left\|x^{*}-x_{\alpha}^{*}\right\|=\lim _{n \rightarrow \infty}\left\|x^{*}-x_{n, \alpha}^{*}\right\|=1-\alpha
$$

and

$$
\left\|x^{*}+x_{\alpha}^{*}\right\|=\lim _{n \rightarrow \infty}\left\|x^{*}+x_{n, \alpha}^{*}\right\|=1+\alpha .
$$

The assumption that the norm is $\mathrm{KK}$ now yields $\lim _{n \rightarrow \infty}\left\|x_{\alpha}^{*}-x_{n, \alpha}^{*}\right\|=0$, for all $\alpha \in A$, which proves our claim.

To finish the proof, note that $l\left(\gamma_{n}\right)<2+n^{-1}$ implies that for all $\alpha_{1}, \alpha_{2} \in A$ and $n \in \mathbf{N}$,

and therefore

$$
\left|\alpha_{1}-\alpha_{2}\right| \leqslant\left\|x_{n, \alpha_{1}}^{*}-x_{n, \alpha_{1}}^{*}\right\| \leqslant\left|\alpha_{1}-\alpha_{2}\right|+n^{-1},
$$

$$
\left\|x_{\alpha_{1}}^{*}-x_{\alpha_{2}}^{*}\right\|=\left|\alpha_{1}-\alpha_{2}\right| \text {. }
$$

The unique continuous extension of this Lipschitz continuous map $\alpha \mapsto x_{\alpha}^{*}$ to $[-1,1]$ now defines a curve $\gamma$ in $S_{X}$ joining the antipodal points $x^{*}=x_{1}^{*}$ and $-x^{*}=x_{-1}^{*}$, obviously with $l(\gamma)=2$, so that flatness has been proved. 
(iii) It remains to prove now that $X^{*}$ cannot be flat if $X$ is WCG. The proof is by transfinite induction on the density character of $X$. Let us first observe that $X$ has an equivalent Fréchet differentiable norm and therefore satisfies all properties of John and Zizler (1974), Theorem 1. Indeed, by Amir and Lindenstrauss (1968) there exists a $w^{*}$-to- $w$ continuous linear injection $T: X^{*} \rightarrow c_{0}(\Gamma)$, for some set $\Gamma$. If $p$ is Day's norm on $c_{0}(\Gamma)$ (see Rainwater (1969)) then $\|\cdot\|_{1}=\|\cdot\|+p T($.$) on X^{*}$ is not only a strictly convex dual norm, so that it arises from a smooth norm $\|\cdot\|_{1}$ on $X$, but also this smooth norm $\left\|_{1}\right\|_{1}$ on $X$ is $\mathrm{KK}$, as one easily checks. Hence $\|.\|_{1}$, being a smooth $\mathrm{KK}$ norm, is Fréchet differentiable. Thus, by John and Zizler (1974), Theorem 1 there exists on $X$ a 'long sequence' of projections $\left\{P_{\alpha}: \omega \leqslant \alpha \leqslant \mu\right\}$ ( $\mu$ is the first ordinal of cardinality dens $X$ ) such that

(1) $P_{\mu}=$ identity, $\left\|P_{\alpha}\right\|=1$ for $\omega \leqslant \alpha \leqslant \mu$,

(2) $P_{\alpha} P_{\beta}=P_{\beta} P_{\alpha}=P_{\min (\alpha, \beta)}$ for $\omega \leqslant \alpha, \beta \leqslant \mu$,

(3) dens $P_{\alpha} X \leqslant \overline{\bar{\alpha}}$ (= cardinality of $\alpha$ ) for $\omega \leqslant \alpha \leqslant \mu$,

(4) for any fixed $x \in X$ the map $\alpha \rightarrow P_{\alpha} x$ is continuous for the order topology and the norm topology, respectively,

(5) the dual projections $P_{\alpha}^{*}$ have the analogous properties $(1)^{*}-(4)^{*}$.

Now, if dens $X=\aleph_{0}$, then, by (3)*, dens $X^{*}=\aleph_{0}$; so $X^{*}$ cannot be flat (Karlovitz (1973)). Next, let $\mathfrak{M}$ be some cardinal $>\aleph_{0}$ and let us suppose that it has been proved for every WCG $X$ with a KK norm and with dens $X<\mathfrak{M}$, that $X^{*}$ is not flat. Let $X$ be a WCG space with a KK norm and with dens $X=\mathfrak{M}$. Let $\mu$ be the first ordinal of cardinality $\mathfrak{M}$ and let $\left\{P_{\alpha}: \omega \leqslant \alpha \leqslant \mu\right\}$ be a long sequence of projections in $X$ as above. Supposing that $X^{*}$ is flat, let $Y \subset X^{*}$ be the (separable) closed linear span of a girth curve in $S_{X^{*}}$. It is then easily derived from (4)* that $Y \subset P_{\alpha}^{*} X^{*}$ for some $\alpha<\mu$. Also $P_{\alpha}^{*} X^{*}$ is isometrically and $w^{*}$-to-w* isomorphic to $\left(P_{\alpha} X\right)^{*}$ by the restriction map. Therefore $P_{\alpha} X$ is a space with a $\mathrm{KK}$ norm and also, of course, WCG. Since dens $P_{\alpha} X<\mathfrak{W}$ the induction hypothesis now implies that $P_{\alpha}^{*} X^{*} \cong\left(P_{\alpha} X\right)^{*}$ is not flat, contradicting $Y \subset P_{\alpha}^{*} X^{*}$. This completes the proof.

CoRollary 3.6. Let $X=\left(\Sigma_{\Gamma} \oplus X_{\gamma}\right)_{l p(\Gamma)}(1<p<\infty)$, or $X=\left(\Sigma_{\Gamma} \oplus X_{\gamma}\right)_{c_{0}(\Gamma)}$, where $\Gamma$ is an arbitrary set, and each $X_{\gamma}(\gamma \in \Gamma)$ is a WCG space with a KK norm. Then neither $X$ nor $X^{*}$ has flat spots.

Proof. It is known (Lindenstrauss (1972)) that $X$ is WCG. Also, since the natural norms on $l^{p}(\Gamma)(1<p<\infty)$ and $c_{0}(\Gamma)$ are $\mathrm{KK}$, it is easily verified that the norm of $X$ is $\mathrm{KK}$ in all cases, se that Proposition 3.5 is applicable.

REMARK 3.7. Corollary 3.6 applies in particular for all choices of finitedimensional $X_{\gamma}$. (Except for $\left(\left(\Sigma_{\Gamma} \oplus X_{\gamma}\right)_{\boldsymbol{l}^{1}(\Gamma)} \cong\left(\left(\Sigma_{\Gamma} \oplus X_{\gamma}^{*}\right)_{c_{0}(\Gamma)}\right)^{*}\right.$ the conclusion then also follows from an obvious modification of Corollary 3.2.) Even in this relatively simple case no direct proof seems available. At any rate the usual arguments do not work (see, for example, Schäffer (1971), Theorem 7). 


\section{Concluding remarks and open problems}

Our study of flat spots, together with Proposition 1.1, has yielded two renorming results for certain non-superreflexive spaces, stated without reference to flat spots, namely Propositions 2.7 and 3.4. The question arises whether they characterize the non-superreflexive spaces among the spaces considered in these propositions. More explicitly, the following problems need solving.

Problem 1. Suppose $X$ is a superreflexive Banach space and that $\left\{x_{n}\right\} \subset S_{X}$ is a weakly convergent sequence, with limit $x_{0} \in S_{X}$. Does it follow that

$$
\underset{n \rightarrow \infty}{\limsup }\left\|x_{0}-x_{n}\right\|<2 ?
$$

Problem 2. Suppose $X$ is a superreflexive Banach space having an FDD with associated sequence of projections $\left\{P_{n}\right\}$. Does it follow that $\liminf _{n \rightarrow \infty}\left\|I-P_{n}\right\|<2$ (hence lim $\sup _{n \rightarrow \infty}\left\|I-P_{n}\right\|<2$ ) for some such F.D.D.?

A final remark concerns Proposition 3.5 (we thank the referee for making this observation). Sometimes the KK norm property for $X$ is defined differently by requiring that on $S_{X} \cdot w^{*}$-convergence for nets implies norm convergence. It is shown in Namioka and Phelps (1975) that spaces $X$ with a KK norm in this stronger sense are SDS (or Asplund spaces) and in Stegall (1979) that for the latter $\boldsymbol{B}_{\boldsymbol{X}^{*}}$ is $\boldsymbol{w}^{*}$-sequentially compact. Thus, if one adopts this stronger definition of a KK norm, the assumption that $B_{X^{*}}$ is $w^{*}$-sequentially compact is not needed in Proposition 3.5. We do not know if (iii) holds without the assumption that $X$ is WCG.

\section{References}

D. Amir and J. Lindenstrauss (1968), "The structure of weakly compact sets in Banach spaces", Ann. of Math. 88, 35-46.

E. Asplund (1968), "Fréchet differentiability of convex functions", Acta Math. 221, 31-48.

E. Bishop and R. R. Phelps (1961), "A proof that every Banach space is subreflexive", Bull. Amer. Math. Soc. 67, 97-98.

D. van Dulst and J. J. Schäffer (1979), "Two examples of flat spots in non-flat Banach spaces", Nieuw Arch. Wisk. (to appear).

P. Enflo (1972), "Banach spaces which can be given an equivalent uniformly convex norm", Israel J. Math. 13, 281-288.

T. Figiel and W. B. Johnson (1974), "A uniformly convex Banach space which contains no $l_{p}$ ", Comp. Math. 29, 179-190.

R. E. Harrell and L. A. Karlovitz (1972), "Non-reflexivity and the girth of spheres, Inequalities III", Acad. Press, 121-127.

R. E. Harrell and L. A. Karlovitz (1974), "The geometry of flat Banach spaces", Trans. Amer. Math. Soc. 192, 209-218. 
R. C. James (1972), "Some self-dual properties of normed linear spaces. Symposium on infinitedimensional topology", Ann. of Math. Studies 69, 159-175.

R. C. James and J. J. Schäffer (1972), "Superreflexivity and the girth of spheres", Israel J. Math. 11, 398-404.

K. John and V. Zizler (1972), "A renorming of dual spaces", Israel J. Math. 12, 331-336.

K. John and V. Zizler (1974), "Smoothness and its equivalents in weakly compactly generated Banach spaces", J. Functional Analysis 15, 1-11.

W. B. Johnson, H. P. Rosenthal and M. Zippin (1971), "On bases, finite-dimensional decompositions and weaker structures in Banach spaces", Israel J. Math. 9, 488-506.

M. I. Kadec (1952), "On the connection between weak and strong convergence", Dopovidi Akad. Nauk Ukraln. RSR 9, 949-952 (Ukrainian).

L. A. Karlovitz (1973), “On the duals of flat Banach spaces”, Math. Ann. 202, 245-250.

L. A. Karlovitz (1976), "On non-expansive mappings", Proc. Amer. Math. Soc. 55, 321-325.

V. Klee (1960/61), "Mappings into normed linear spaces", Fund. Math. 49, 25-34.

J. Lindenstrauss (1972), "Weakly compact sets-their topological properties and the Banach spaces they generate", Ann. of Math. Studies 69, 235-273.

J. Lindenstrauss and H. P. Rosenthal (1969), "The $\mathscr{L}_{p}$ spaces", Israel J. Math. 7, 325-349.

I. Namioka and R. R. Phelps (1975), "Banach spaces which are Asplund spaces", Duke Math.J. 42, 735-750.

A. J. Pach (1979), "Flat spots and super-reflexivity in normed spaces (preprint, University of Amsterdam).

J. Rainwater (1969), "Local uniform convexity of Day's norm on $c_{0}(\Gamma)$ ", Proc. Amer. Math. Soc. 22, 335-339.

J. J. Schäffer (1967), "Inner diameter, perimeter and girth of spheres", Math. Ann. 173, 59-79.

J. J. Schäffer (1971), "On the geometry of spheres in L-spaces", Israel J. Math. 10, 114-120.

J. J. Schäffer (1976), Geometry of spheres in normed spaces (Lecture notes in pure and applied mathematics, Marcel Dekker, New York and Basel).

C. Stegall (1977), "The Radon-Nikodym property in conjugate spaces II" (preprint).

S. Troyanski (1971), "On locally uniformly convex and differentiable norms in certain nonseparable Banach spaces”, Studia Math. 37, 173-180.

B. S. Tsirelson (1974), "Not every Banach space contains $l_{p}$ or $c_{0}$ ", Functional Anal. Appl. 8, 138-141 (translated from Russian).

Mathematisch Instituut

Universiteit van Amsterdam

Roetersstraat 15, Amsterdam

Holland 